\title{
Tratamiento farmacológico actual del tromboembolismo pulmonar
}

\author{
Limberth Machado-Villarroel, *凶 Gregoria Gómez-Hernández* \\ *Especialista en Medicina Crítica y Cardioneumología. Hospital Ángeles Cd. Juárez y Universidad Autónoma de Ciudad Juárez UACJ; \\ ${ }^{\ddagger}$ Medicina interna. Instituto Nacional de Ciencias Médicas y Nutrición Salvador Zubirán, Ciudad de México. \\ Trabajo recibido: 10-IV-2013; aceptado: 21-XI-2013
}

\begin{abstract}
RESUMEN. La enfermedad tromboembólica venosa representa un espectro de una misma entidad que incluye al tromboembolismo pulmonar y la trombosis venosa profunda; ambas se originan como complicación de diversas patologías. La anticoagulación con heparina convencional o no fraccionada y heparina de bajo peso molecular durante 5-10 días es el tratamiento inicial de elección. El tratamiento del tromboembolismo pulmonar se continúa habitualmente con antagonistas de la vitamina K entre 3 y 6 meses; en algunos casos, la anticoagulación es hasta por 1 año debido a la latencia de factores de riesgo. Existen varios estudios que han confirmado la efectividad y seguridad de las heparinas de bajo peso molecular comparadas con la heparina no fraccionada en el tratamiento de la fase aguda del tromboembolismo pulmonar. Hoy en día el uso de esta última es de rutina, tanto en la prevención como en el tratamiento de la trombosis venosa profunda. Esto difiere del tratamiento del tromboembolismo pulmonar masivo y/o submasivo, que requiere tratamiento fibrinolítico debido a que la mortalidad alcanza hasta el $70 \%$, fuertemente relacionada con falla ventricular derecha e insuficiencia cardiaca.
\end{abstract}

Palabras clave: Enfermedad tromboembólica venosa, tromboembolismo pulmonar, heparina no fraccionada, heparina de bajo peso molecular, antagonistas de la vitamina $\mathrm{K}$.

\begin{abstract}
Venous thromboembolism represents a spectrum of the same entity which includes pulmonary embolism and deep vein thrombosis. The conventional anticoagulation with unfractionated heparin and low-molecular-weight heparin for 5-10 days is the initial treatment of choice. Pulmonary embolism treatment typically continues with vitamin $\mathrm{K}$ antagonists between 3 and 6 months, in some cases anticoagulation is up to 1 year, due to the latency of risk factors. Several studies have confirmed the effectiveness and safety of low-molecular-weight heparin compared with unfractionated heparin in the acute phase treatment of pulmonary embolism; nowadays the use of the latter is routine in both prevention and treatment of deep-vein thrombosis. This differs from the treatment of massive and/or submassive pulmonary embolism requiring fibrinolytic therapy because mortality is up to $70 \%$, strongly related to right ventricular failure and heart failure.
\end{abstract}

Key words: Venous thromboembolic disease, pulmonary thromboembolism, unfractionated heparin, low-molecular-weight heparin, vitamin $\mathrm{K}$ antagonists.

\section{INTRODUCCIÓN}

El tromboembolismo pulmonar (TEP) es la complicación más grave de la trombosis venosa profunda (TVP). Ambas son manifestaciones diferentes de una misma enfermedad: la enfermedad tromboembólica venosa (ETV). Este concepto es fundamental para la terapéutica de la enfermedad, ya que de los pacientes con TVP que desarrollan síntomas de TEP, el $40 \%$ realmente lo tiene. La evidencia actual sugiere que todo paciente con bajo riesgo para mortalidad o eventos cardiovasculares adversos por TEP menor, sin disfunción del ventrículo derecho (DVD) y con perfusión sistémica normal, debe recibir heparina y anticoagulación oral. ${ }^{1} \mathrm{Si}$ el riesgo de mortalidad es alto por TEP masivo o mayor, hipertensión arterial pulmonar (HAP) grave y DVD, entonces debe intentarse una estrategia de reperfusión farmacológica o mecánica; en algunos casos, una estrategia de reperfusión combinada mejora la evolución. ${ }^{2}$

Considerando que en la fisiopatología el grado de obstrucción de la circulación pulmonar determina el grado de las alteraciones cardiopulmonares, los objetivos del tratamiento farmacológico son: lisar el trombo, disminuir los efectos adversos como la HAP, así como la recurrencia y mortalidad. ${ }^{3}$ En ocasiones y debido al tratamiento pueden presentarse complicaciones hemorrágicas; el tratamiento óptimo es aquel que reduce estas complicaciones agudas; no obtante, a largo plazo, el objetivo es también reducir recidivas, complicaciones crónicas como la hipertensión pulmonar e insuficiencia 
cardíaca. Cuando el TEP es masivo, con compromiso hemodinámico, la terapia de reperfusión con fibrinolíticos es la de elección para reperfundir con prontitud la circulación vascular pulmonar, con restablecimiento de la hemodinamia.

El propósito de este artículo de revisión es describir el tratamiento anticoagulante en pacientes con TEP, tanto con heparina no fraccionada (HNF), heparinas de bajo peso molecular (HBPM), anticoagulantes orales, así como mencionar las nuevas opciones de tratamiento y el tratamiento trombolítico.

\section{HEPARINA NO FRACCIONADA}

En estudios comparativos desde 1960 se demostraron los beneficios de la HNF en comparación con pacientes sin tratamiento. ${ }^{4}$ La HNF disminuye fenómenos de retrombosis al inhibir la trombina circulante, pero no actúa sobre la trombina ligada al trombo; impide la agregación plaquetaria y de la fibrina ligada al trombo, con lo que se evita su crecimiento y permite que la fibrinólisis endógena actúe in vivo. La HNF y la HBPM son la piedra angular en el tratamiento del TEP; sin embargo, no hay evidencia que demuestre una mejor evolución en

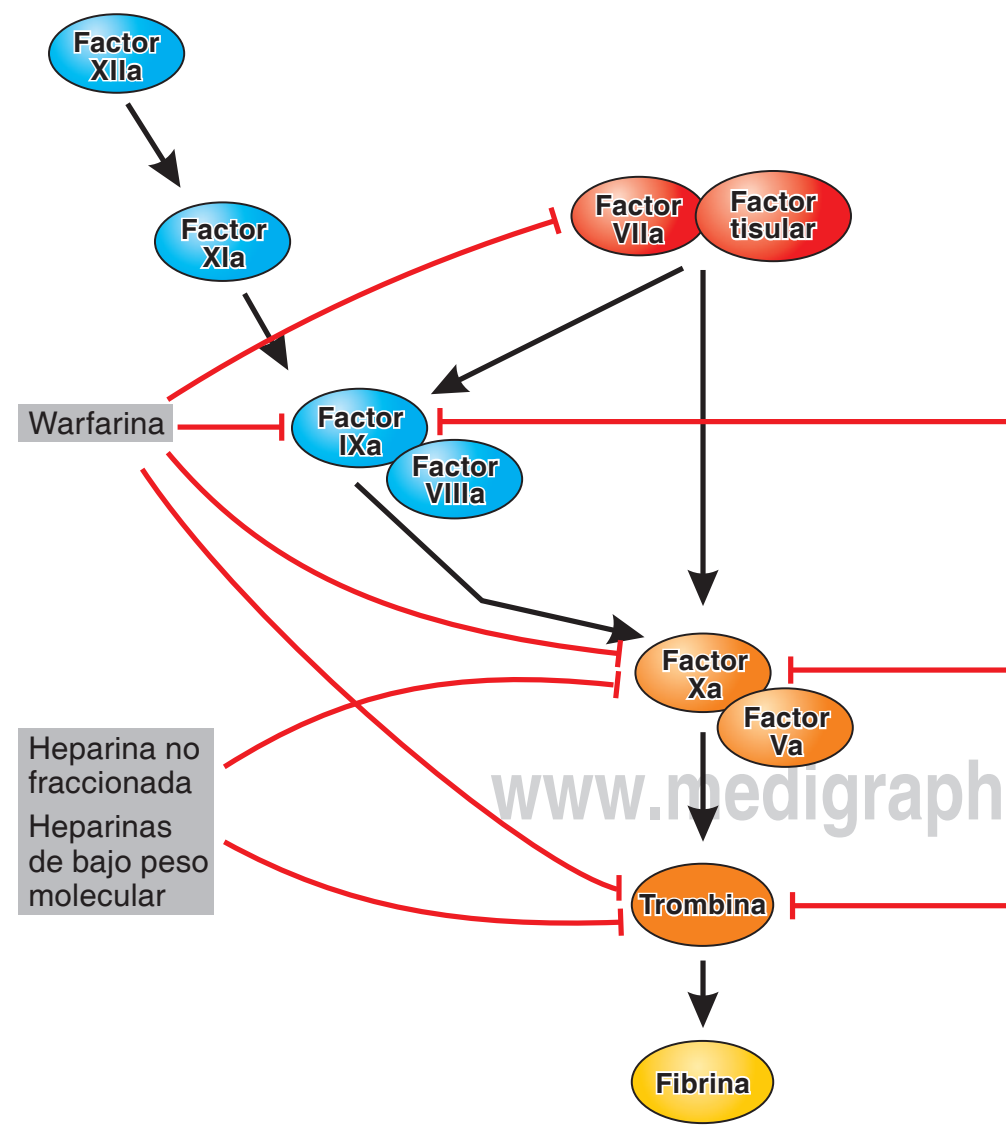

presencia de DVD. ${ }^{3}$ El tratamiento debe iniciarse con anticoagulantes por vía parenteral, como es la HNF. En algunos casos debe estar siempre considerada, aun en pacientes con sospecha de embolismo pulmonar, mientras se espera la confirmación diagnóstica. ${ }^{5}$

Hoy, la HNF es considerada como la elección en el tratamiento de la fase aguda del TEP estable. La característica bioquímica la compone una mezcla heterogénea de cadenas de polisacáridos. Su peso molecular oscila entre 3,000 y 30,000 Dalton, con una media de 12,000 a 16,000 Dalton; la HNF actúa como anticoagulante, su efecto terapéutico se basa en una acción antitrombina al unirse a la antitrombina III plasmática, inactivando diferentes factores de la coagulación, entre los que también se incluye el factor $\mathrm{Xa}^{2,6}$ (figura 1). La dosis se ajusta al peso del paciente, de $80 \mathrm{U} / \mathrm{kg}$ como bolo inicial, y continúa con una infusión a razón de 18 $\mathrm{U} / \mathrm{kg} / \mathrm{h}$. La dosis debe modificarse de acuerdo con el tiempo parcial de tromboplastina (TPT); el objetivo es mantener entre 1.5 y 2.5 veces el control. El monitoreo del TPT debe realizarse cada 4-6 horas, y 3 horas después de cada ajuste de dosis o diariamente si la dosis terapéutica está en rango ${ }^{5,7}$ (tabla 1). Un manejo y un seguimiento adecuados reducen la mortalidad y,
Aptámero

de RNA
Figura 1.

Mecanismo de acción de los fármacos anticoagulantes. Ref. 43. Adapted by permission from Macmillan Publishers Ltd: Nature 451:914-918, copyright 2008.
Fondaparinux Idraparinux pixabán Rivaroxabán Desirudina Dabigatrán 
Tabla 1. Dosificación de heparina no fraccionada por vía intravenosa basada en el tiempo parcial de tromboplastina. ${ }^{44}$

\begin{tabular}{ll}
\hline TPT & Modificación \\
\hline$<35 \mathrm{~s}(<1.2$ veces el control) & $80 \mathrm{U} / \mathrm{kg}$ bolo e incrementar la infusión a razón de $4 \mathrm{U} / \mathrm{kg} / \mathrm{h}$ \\
$35-45 \mathrm{~s}(1.2-1.5$ veces el control) & $40 \mathrm{U} / \mathrm{kg}$ bolo e incrementar la infusión a razón de $2 \mathrm{U} / \mathrm{kg} / \mathrm{h}$ \\
$46-70 \mathrm{~s}(1.5-2.3$ veces el control) & No modificar \\
$71-90 \mathrm{~s}(2.3-3.0$ veces el control) & Reducir infusión a razón de $2 \mathrm{U} / \mathrm{kg} / \mathrm{h}$ \\
$>90 \mathrm{~s}(>3.0$ veces el control) & Detener la infusión por una hora y, posteriormente, reducir la \\
& infusión a razón de $3 \mathrm{U} / \mathrm{kg} / \mathrm{h}$ \\
\hline
\end{tabular}

TEP: tromboembolismo pulmonar.

sobre todo, las recidivas en pacientes con TEP. Es recomendable mantener el tratamiento con heparina por al menos 5-7 días, más en casos de embolia pulmonar masiva. Entre los efectos secundarios más importantes está la hemorragia, hasta en el $20 \%$ de los $\operatorname{casos}^{8,9} \mathrm{En}$ caso de complicaciones hemorrágicas, el antídoto de la heparina es el sulfato de protamina, $1 \mathrm{mg}$ de protamina antagoniza $100 \mathrm{U}$ de heparina recién administrada; el tratamiento subsecuente con otros anticoagulantes puede permanecer por más de 3 meses. Durante los siguientes días de iniciado el tratamiento con heparina, también pueden presentarse complicaciones como la trombocitopenia, por lo que debe realizarse un recuento de plaquetas cada 24 a 48 horas para descartar esta complicación. ${ }^{10}$ Cuando el tratamiento no es óptimo, un predictor de recurrencia del TEP son las propias características clínicas del primer evento. ${ }^{11,12}$

\section{HEPARINAS DE BAJO PESO MOLECULAR}

La HBPM es una molécula de 4,000-5,000 Dalton; existe un número importante de este grupo de medicamentos extensamente estudiados, así como también las preparaciones de pentasacáridos. Estos medicamentos fueron descubiertos debido a que los efectos anticoagulantes de la HNF dependen particularmente de un pentasacárido que presenta una elevada afinidad por la antitrombina; mediante métodos enzimáticos o químicos se fraccionaron las cadenas de la HNF en búsqueda de fragmentos con mejor actividad antitrombótica y menor efecto de hemorragia. Las fracciones de la HBPM pierden su capacidad para alargar el tiempo de tromboplastina parcial activada, pero mantienen la capacidad de inhibir el factor $X$ activado. $^{6}$

Existen diferentes moléculas de HBPM; en México están disponibles las siguientes: dalteparina, enoxaparina, tinzaparina y nadroparina. Los estudios farmacocinéticos de las HBPM muestran que tienen una biodisponibilidad superior después de la administración por vía subcutánea y alcanzan el $90 \%$ del equivalente a la forma intravenosa; esta característica farmacocinética es debida al tamaño de las moléculas, lo que facilita la difusión a través de los capilares del tejido subcutáneo. Otra característica biológica de las HBPM es que presentan una vida media más prolongada en su actividad anticoagulante (medida por la actividad antiXa). Actualmente se consideran como Clase I, nivel de evidencia A en el tratamiento del TEP. ${ }^{13}$ La HBPM puede iniciarse tan pronto como se realice el diagnóstico. ${ }^{14,15}$ Varios estudios compararon la eficacia y seguridad de la HBPM y la HNF. El metaanálisis más importante, con 1951 pacientes con embolismo pulmonar sintomático o asintomático y TVP sintomático, demostró que las HBPM son tan eficaces como la HNF debido a que no se asociaron a mayor recurrencia (OR 0.63; IC 95\%, 0.33-1.18), sangrados mayores (OR 0.67; IC 95\%, 0.36-1.27) ni causas de mortalidad (OR 1.20; IC 95\%, 0.592.45)..$^{16}$ Entre las ventajas que han demostrado están: adecuada biodisponibilidad, predicción de la dosis, aplicación subcutánea, habitualmente sin requerir monitoreo y bajo riesgo de trombocitopenia. ${ }^{8,17}$ Estas características, en conjunto, proporcionan una respuesta terapéutica predecible, con menos variaciones individuales, lo que hace posible su empleo a dosis fijas en función del peso corporal del paciente una o dos veces al día (tabla 2). En algunos casos, se puede determinar la concentración plasmática midiendo la actividad antiXa de las HBPM a las cuatro horas de haber administrado el fármaco. En pacientes con falla renal debe existir precaución y ajustarse, si es posible, de acuerdo con el nivel de antiXa. En ocasiones, la HNF se prefiere de manera inicial en pacientes con insuficiencia renal grave (depuración de creatinina < $30 \mathrm{~mL} / \mathrm{min}$ ) debido a que su eliminación no es por vía renal. Posteriormente la HNF puede remplazarse por HBPM por vía subcutánea ajustando al peso y a la depuración. ${ }^{5}$ Datos recientes sugieren que los niveles del dímero-D pueden ayudar a determinar el grado de anticoagulación y la decisión de mantenerla. En la actualidad, y durante la fase inicial del TEP, se cuenta con la aprobación del uso de enoxaparina, tinzaparina, fondaparina, dalteparina. ${ }^{5,18}$ 
Tabla 2. Régimen de heparina de bajo peso molecular aprobado para el tratamiento del tromboembolismo pulmonar. ${ }^{45}$

\begin{tabular}{lcc}
\hline HBPM & Dosis & Intervalo \\
\hline Enoxaparina & $1.0 \mathrm{mg} / \mathrm{kg}$ & cada $12 \mathrm{~h}$ \\
& $1.5 \mathrm{mg} / \mathrm{kg}$ & cada $24 \mathrm{~h}$ \\
Tinzaparina & $175 \mathrm{U} / \mathrm{kg}$ & cada $24 \mathrm{~h}$ \\
Fondaparinux & $5 \mathrm{mg}($ peso $<50 \mathrm{~kg})$ & cada $24 \mathrm{~h}$ \\
& $7.5 \mathrm{mg}($ peso $50-100 \mathrm{~kg})$ & cada $24 \mathrm{~h}$ \\
& $10 \mathrm{mg}(>100 \mathrm{~kg})$ & cada $24 \mathrm{~h}$ \\
\hline
\end{tabular}

En pacientes con cáncer, la dalteparina está aprobada para tratamiento extendido de ETV, TVP y/o TEP, con una dosis inicial de 200 U/kg s.c. La dosis diaria de enoxaparina de $1.5 \mathrm{mg} / \mathrm{kg}$ está aprobada como tratamiento de TEP en pacientes hospitalizados en Estados Unidos y en algunos países europeos.

Abreviaturas: ETV: Enfermedad tromboembólica; TVP: trombosis venosa profunda; TEP: tromboembolismo pulmonar.

\section{ANTICOAGULANTES ORALES}

Los cumarínicos son fármacos derivados de la 4-hidroxicumarina, y su acción tiene efecto antagónico sobre la vitamina $\mathrm{K}$, ocasionando disminución de la actividad de los factores de coagulación dependientes de ésta (II, VII, IX y X), así como de las proteínas C y S. Dentro de este grupo se encuentran el cumarol, acenocumarol y warfarina, medicamentos que pueden iniciarse el primer día de tratamiento. ${ }^{7,19}$ Habitualmente, al inicio deben administrarse junto a la heparina por lo menos 48 horas hasta obtener un efecto terapéutico deseado. Después, deben medirse controles de coagulación, particularmente el tiempo de protrombina, para más tarde establecer la continuidad de los anticoagulantes orales. ${ }^{20}$ Entre las limitaciones del tratamiento con anticoagulantes orales están el estrecho margen terapéutico de sobredosificación, la sensibilidad y variabilidad de sus niveles plasmáticos - los cuales pueden modificarse por el grado de absorción-, la interacción con múltiples medicamentos e, inclusive, la dieta. ${ }^{6}$ En conjunto, todos estos factores producen inestabilidad del INR (del inglés International Normalized Ratio), con impacto en la terapéutica. ${ }^{21,22} \mathrm{EI}$ INR es un método que permite estandarizar los valores obtenidos de la anticoagulación y monitorizar la anticoagulación por vía oral. ${ }^{20}$ Otro inconveniente es el hecho de que al suspender el tratamiento, la normalización de la coagulación no es inmediata. Estos fenómenos obligan a mantener un control estricto en los pacientes tratados con estos fármacos. ${ }^{23}$ El factor más importante de predicción de sangrado es, en efecto, la intensidad de anticoagulación. Si el paciente presenta niveles mayores de anticoagulación, en caso de prolongación excesiva del INR, es necesario administrar vitamina K1 (3 mg). ${ }^{24}$ En estos casos, a veces es necesario aportar los factores de coagulación en forma de plasma fresco o concentrados de crioprecipitados hasta alcanzar un valor de INR inferior a $1,5 .{ }^{7}$ Debido a lo anterior, debe haber extremo cuidado con los anticoagulantes orales, quizá contraindicados en aquellos con pobre apego al tratamiento o que no tengan la posibilidad de asumir y acudir a los controles durante el tiempo que dure su tratamiento; éste es el grupo de enfermos en el que las HBPM puedan ser más apropiadas.

\section{INHIBIDORES DE LA TROMBINA}

En los últimos años fueron identificados nuevos anticoagulantes orales denominados «inhibidores directos de la trombina», como la hirudina. Éste es el primer grupo de medicamentos con mínimas complicaciones hemorrágicas y menor riesgo de desarrollar trombocitopenia inducida. ${ }^{25}$ Dentro de este grupo está el dabigatrán, fármaco que promete un desempeño importante en el futuro. ${ }^{26,27} \mathrm{En}$ el estudio realizado por Schulman et al. para el tratamiento a largo plazo del TEP, se comparó al dabigatrán contra los antagonistas de la vitamina K; se observó que los pacientes con TEP sintomático no tuvieron diferente respuesta a dabigatrán que a los antagonistas de la vitamina $\mathrm{K}$ en términos de recurrencia del TEP o sangrado. ${ }^{28}$ Uno de los medicamentos que ha reportado buenos resultados es el inhibidor directo del factor Xa, el rivaroxabán, que ha sido aprobado para su uso clínico en varios países y ha demostrado su beneficio. ${ }^{29}$ En el estudio Einstein-Extension se incluyeron pacientes con TVP o TEP después de un período inicial de anticoagulación (antagonistas de vitamina $\mathrm{K}$ versus rivaroxabán por 6 o 12 meses), y compararon a rivaroxabán contra placebo para tratamiento de seguimiento. Dentro de los resultados, en el análisis del subgrupo de los pacientes con TEP, éstos tuvieron una respuesta similar tanto al rivaroxabán como a antagonistas de la vitamina K en términos de recurrencia de ETV o sangrado. ${ }^{30}$ En otro estudio que incluyó a pacientes con TEP sintomático, se comparó al rivaroxabán contra el tratamiento convencional (enoxaparina seguida de antagonistas de la vitamina K) por 3, 6 o 12 meses. Se observó recurrencia de ETV en $2.1 \%$ en el grupo de rivaroxabán 
contra $1.8 \%$ en el grupo del tratamiento convencional (HR 1.12; IC 95\%, 0.75-1.68; $p=0.003$ ) y $1.1 \%$ eventos de sangrado mayor contra $2.2 \%$, respectivamente (HR 0.49; IC 95\%, 0.31-0.79; $p=0.003$ ); por lo que se concluyó que el tratamiento inicial y a largo plazo con rivaroxabán no fue inferior al tratamiento convencional, con probablemente un mejor perfil de seguridad. ${ }^{31}$ La $9^{a}$ edición de los lineamientos de la ACCP enfocados en el tratamiento antitrombótico emite una recomendación débil sobre el uso de dabigatrán o rivaroxabán en la ETV. La justificación está centrada en la falta de estudios de seguridad fase IV (postcomercialización). ${ }^{32}$

\section{TRATAMIENTO TROMBOLÍTICO}

Está dirigido para embolismo pulmonar submasivo y masivo con inestabilidad hemodinámica donde, en estos casos, es necesaria la aplicación conjunta de heparina y fibrinolíticos. El objetivo de esta intervención es permeabilizar las arterias pulmonares para mejorar las condiciones hemodinámicas, evitar consecuencias de disfunción ventricular derecha e hipertensión pulmonar crónica, además de intervenir también sobre la TVP. Sin embargo, el uso de estos medicamentos puede llevar al paciente a serias complicaciones, por lo cual están establecidas claramente las contraindicaciones de su aplicación ${ }^{2,33}$ (tabla 3). Las complicaciones hemorrágicas son las más preocupantes; ocurren en el 14\% de los pacientes con TEP que recibieron fibrinólisis posterior a la angiografía pulmonar; $;^{34,35}$ el principal origen del sangrado es el sitio de punción para la angiografía, que ocasiona sangrados mayores en el 36 al 45\%.36,37 Otra complicación que se presenta es la hemorragia intracraneal, en el $1.9 \% .^{38}$ Las complicaciones hemorágicas en pacientes a quienes se les diagnosticó TEP de manera no invasiva y se sometieron a tratamiento fibrinolítico, hasta el momento no han reportado san- grados mayores. ${ }^{38}$ Otras complicaciones que pueden aparecer son las reacciones alérgicas, más frecuentes con la estreptoquinasa y urokinasa. El rTPA es considerado como el de menor riesgo de sangrado. Existen varios estudios que comparan la eficacia y el beneficio de estos fármacos. En un estudio quedó demostrado que la trombólisis, al permeabilizar las arterias pulmonares, mejora los parámetros hemodinámicos con un incremento del $80 \%$ en el IC y una reducción del $40 \%$ de la hipertensión pulmonar después de la aplicación de estreptoquinasa. ${ }^{7,39}$ Recién, un estudio multicéntrico mostró que el rTPA reducía el $12 \%$ de la obstrucción vascular después de dos horas de haber recibido la infusión; estos cambios no se mostraron en pacientes que recibían heparina. ${ }^{40}$

La mayoría de los trombolíticos tienen un mecanismo de acción semejante a los del sistema de fibrinólisis natural del organismo. De manera natural, el plasminógeno es activado por los Pas (activadores del plasminógeno), entre ellos la serina proteasa uPA (activador del plasminógeno tipo urocinasa) y tPA (activador del plasminógeno tipo tisular); éstos efectúan una proteólisis parcial de la proteína para convertirla en plasmina activa. Todos lo trombolíticos actúan también transformando el plasminógeno (proenzima inactiva) en plasmina, sustancia bioquímicamente potente que degrada componentes del trombo, se une a la red de fibrina (polímero insoluble), procediendo a su proteólisis y transformando la malla de fibrina en pequeños restos proteicos llamados productos de degradación de la fibrina (productos solubles); los trombolíticos difieren en cuanto a su capacidad de activación del plasminógeno plasmático o unido a fibrina (trombolíticos fibrino-selectivos y no selectivos). El activador tisular del plasminógeno recombinante (rTPA) es el más utilizado en la actualidad para este efecto, debido a la comodidad en su administración, su eficacia y menores complica-

Tabla 3. Contraindicaciones de la terapia fibrinolítica. ${ }^{46}$

\begin{tabular}{ll}
\hline Contraindicaciones absolutas & Contraindicaciones relativas \\
\hline - Enfermedad vascular cerebral o EVC de origen no determinado & - Ataque isquémico transitorio en los 6 meses previos \\
- EVC en los 6 meses previos & - Terapia anticoagulante oral \\
- Neoplasia del sistema nervioso central & - Embarazo o dentro de la primera semana de posparto \\
- Trauma mayor reciente/cirugía/lesión craneal (dentro las 3 & - Punciones no compresibles \\
- Semanas previas) & - RCP \\
- Hemorragia digestiva en el último mes & - Enfermedán refractaria (presión sistólica de 180 mph) \\
& - Endocarditis infecciosa
\end{tabular}

Abreviaturas: EVC: Enfermedad vascular cerebral; RCP: Reanimación cardiopulmonar. 
Tabla 4. Terapia trombolítica aprobada para tromboembolia pulmonar. ${ }^{45}$

Estreptoquinasa 250,000 IU en $30 \mathrm{~min}$, seguidas de $100,000 \mathrm{IU} / \mathrm{h}$ durante $12-24 \mathrm{~h}$

Régimen acelerado: 1.5 millón UI en $2 \mathrm{~h}$

Urokinasa 4,400 IU/kg dosis en $10 \mathrm{~min}$, completar con

4,400 IU/kg/h durante 12-24 h

Régimen acelerado: 3 millón UI en $2 \mathrm{~h}$

rtPA $100 \mathrm{mg}$ en $2 \mathrm{~h}$ en total, $0.6 \mathrm{mg} / \mathrm{kg}$ o $10 \mathrm{mg}$ en $15 \mathrm{~min}$ y $90 \mathrm{mg}$ en $2 \mathrm{~h}$

ciones (tabla 4). La eficacia de la fibrinólisis puede ser evaluada con la medición de la saturación arterial de oxígeno en sangre venosa mixta o con el gradiente de presión parcial de bióxido de carbono $\left(\mathrm{CO}_{2}\right)$ arterial y la presión de $\mathrm{CO}_{2}$ en el alvéolo $\left(\mathrm{ETCO}_{2}\right) \mathrm{PaCO}_{2} / \mathrm{ETCO}_{2}{ }^{41}$ Los fibrinolíticos se administran por vía sistémica y en ocasiones también puede ser de manera directa en la arteria pulmonar. Leeper et al. emplearon estreptoquinasa intraarterial pulmonar en el tratamiento de pacientes con TEP. ${ }^{42}$ Asimismo, cabe recalcar que además del tratamiento farmacológico en estos pacientes, es importante el soporte hemodinámico y de oxigenoterapia que debe ofrecerse al paciente durante el tratamiento y la recuperación.

\section{CONCLUSIÓN}

Al presente, el tratamiento médico del TEP se basa en el uso de HNF, HBPM; su administración ante la sospecha de TEP debe iniciarse sin retraso alguno, considerando siempre los efectos colaterales. En pacientes con insuficiencia renal se prefiere la HNF antes del uso de la HBPM; una vez iniciado el tratamiento, otro aspecto importante es la continuidad de la anticoagulación con antagonistas de vitamina K; la duración de la terapia dependerá de los factores de riesgo que favorecen la ETV. El tratamiento trombolítico es el tratamiento de primera línea en pacientes con choque cardiogénico e hipotensión persistente. El uso de los trombolíticos en pacientes con TEP moderado o sin alto riesgo no se recomienda, pero puede considerarse en casos seleccionados. En pacientes de bajo riesgo no es recomendable el uso de trombolíticos. El advenimiento de nuevas terapias como los inhibidores de la trombina y factor $\mathrm{X}$ activado amplía las perspectivas y promete mejores resultados en un futuro cercano.

\section{REFERENCIAS}

1. Moser KM, Fedullo PF, LitteJohn JK, Crawford R. Frequent asymptomatic pulmonary embolism in patients with deep venous thrombosis. JAMA 1994;271(3):223225.

2. Kasper W, Konstantinides S, Geibel A, et al. Management strategies and determinants of outcome in acute major pulmonary embolism: results of a multicenter registry. J Am Coll Cardiol 1997;30(5):1165-1171.

3. Jerjes-Sánchez C, Elizalde GJJ, Sandoval ZJ, et al. Diagnóstico, estratificación y tratamiento de la tromboembolia pulmonar aguda. Guías y recomendaciones del Capítulo de Circulación Pulmonar de la Sociedad Mexicana de Cardiología. Arch Cardiol Mex 2004;74(3 Supl): S547-S585.

4. Barritt DW, Jordan SC. Anticoagulant drugs in the treatment of pulmonary embolism. A controlled trial. Lancet 1960;1(7138):1309-1312.

5. Task Force on Pulmonary Embolism, European Society of Cardiology. Guidelines on diagnosis and management of acute pulmonary embolism. Eur Heart J 2000;21(16):1301-1336.

6. Ruiz MJ, Andreo GM. Tratamiento médico del tromboembolismo pulmonar. En: Juretschke MA, Barbosa AC, editores. Tromboembolismo pulmonar. Monografías NEUMOMADRID. Vol. IV. España: NEUMOMADRID, Doyma; 2002.p.111-130.

7. Tapson VF. Acute pulmonary embolism. N Engl J Med 2008;358(10):1037-1052.

8. Douketis JD, Kearon C, Bates S, Duku EK, Ginsberg JS. Risk of fatal pulmonary embolism in patients with treated venous thromboembolism. JAMA 1998;279(6):458-462.

9. Conti S, Daschbach M, Blaisdell FW. A comparison of high-dose versus conventional dose heparin therapy for deep vein thrombosis. Surgery 1982;92(6):972-980.

10. Warkentin TE, Kelton JG. A 14-year study of heparin induced thrombocytopenia. Am J Med 1996;101(5):502507.

11. Villagómez A, Alcántar E, Avelar F, et al. Guías para el diagnóstico, prevención y tratamiento de la enfermedad tromboembólica venosa. Med Inter Mex 2006;22:S1-S37.

12. Kearon C. Long-term management of patients after venous thromboembolism. Circulation 2004;110(9 Suppl 1):110-18.

13. Ageno W, Gallus AS, Wittkowsky A, Crowther M, Hylek EM, Palareti G; American College of Chest Physicians. Oral anticoagulant therapy: Antithrombotic therapy and prevention of thrombosis, 9th ed: American College of Chest Physicians Evidence-Based Clinical Practice Guidelines. Chest 2012;141(2 Suppl):e44S-e88S.

14. Hyers TM, Hull RD, Weg JG. Antithrombotic therapy for venous thromboembolic disease. Chest 1995;108(4 Suppl):335S-351S.

15. Remy-Jardin M, Pistolesi M, Goodman LR, et al. Management of suspected acute pulmonary embolism in the era of CT angiography: a statement from the Fleischner Society. Radiology 2007;245(2):315-329.

16. Quinlan DJ, McQuillan A, Eikelboom JW. Low-molecularweight heparin compared with intravenous unfractionated heparin for treatment of pulmonary embolism: a meta analysis of randomized, controlled trials. Ann Intern Med 2004;140(3):175-183. 
17. Jaff MR, McMurtry MS, Archer SL, et al; American Heart Association Council on Cardiopulmonary, Critical Care, Perioperative and Resuscitation; American Heart Association Council on Peripheral Vascular Disease; American Heart Association Council on Arteriosclerosis, Thrombosis and Vascular Biology. Management of massive and submassive pulmonary embolism, iliofemoral deep vein thrombosis, and chronic thromboembolic pulmonary hypertension: a scientific statement from the American Heart Association. Circulation 2011;123(16):1788-1830.

18. Garcia DA, Baglin TP, Weitz Jl, Samama MM; American College of Chest Physicians. Parenteral anticoagulants: Antithrombotic therapy and prevention of thrombosis, 9th ed: American College of Chest Physicians EvidenceBased Clinical Practice Guidelines. Chest 2012;141(2 Suppl):e24S-e43S.

19. Brandjes DP, Heijboer $H$, Büller HR, de Rijk $M$, Jagt $H$, ten Cate JW. Acenocoumarol and heparin compared with acenocoumarol alone in the initial treatment of proximal-vein thrombosis. N Engl J Med 1992;327(21):14851489.

20. Fasco MJ, Hildebrandt EF, Suttie JW. Evidence that warfarin anticoagulant action involves two distinct reductase activities. J Biol Chem 1982;257(19):11210-11212.

21. Sconce E, Khan T, Mason J, Noble F, Wynne H, Kamali F. Patients with unstable control have a poorer dietary intake of vitamin $K$ compared to patients with stable control of anticoagulation. Thromb Haemost 2005;93(5):872-875.

22. Kurnik D, Loebstein R, Rabinovitz H, Austerweil N, Halkin $\mathrm{H}$, Almog S. Over-the-counter vitamin K1-containing multivitamin supplements disrupt warfarin anticoagulation in vitamin K1-depleted patients. A prospective, controlled trial. Thromb Haemost 2004;92(5):1018-1024.

23. Choonara IA, Malia RG, Haynes BP, et al. The relationship between inhibition of vitamin K1 2,3-epoxide reductase and reduction of clotting factor activity with warfarin. Br J Clin Pharmacol 1988;25(1):1-7.

24. Forfar JC. Prediction of hemorrhage during long-term oral coumarin anticoagulation by excessive prothrombin ratio. Am Heart J 1982;103(3):445-446.

25. Toschi V, Lettino M, Gallo R, Badimon JJ, Chesebro JH. Biochemistry and biology of hirudin. Coron Artery Dis 1996;7(6):420-428.

26. Lewis BE, Walenga JM, Wallis DE. Anticoagulation with Novastan (argatroban) in patients with heparin-induced thrombocytopenia and heparin-induced thrombocytopenia and thrombosis syndrome. Semin Thromb Hemost 1997;23(2):197-202.

27. Connolly SJ, Ezekowitz MD, Yusuf S, et al.; RE-LY Steering Committee and Investigators. Dabigatran versus warfarin in patients with atrial fibrillation. N Engl J Med 2009;361(12):1139-1151.

28. Schulman S, Kearon C, Kakkar AK, et al.; RE-COVER Study Group. Dabigatran versus warfarin in the treatment of acute venous thromboembolism. N Engl J Med 2009;361(24):2342-2352.
29. Vargas RAG, Ramírez LAN, Medina VME. Nuevos anticoagulantes: dabigatrán, rivaroxabán y apixabán. Gac Méd Méx 2012;148(3):257-264.

30. Bauersachs R, Berkowitz SD, Brenner B, et al.; EINSTEIN Investigators. Oral rivaroxaban for symptomatic venous thromboembolism. N Engl J Med 2010;363(26):24992510.

31. Büller HR, Prins $M H$, Lensin AW, et al.; EINSTEIN-PE investigadores. Oral rivaroxaban for the treatment of symptomatic pulmonary embolism. N Engl J Med 2012;366(14):1287-1297.

32. Kearon C, AkI EA, Comerota AJ, et al.; American College of Chest Physicians. Antithrombotic therapy for VTE disease: Antithrombotic therapy and prevention of thrombosis, 9th ed: American College of Chest Physicians Evidence-Based Clinical Practice Guidelines. Chest 2012;141(2 Suppl):e419S-494S.

33. Arcasoy SM, Kreit JW. Thrombolytic therapy of pulmonary embolism: a comprehensive review of current evidence. Chest 1999;115(6):1695-1707.

34. Monreal M, Casals A, Las Heras G, et al. Hemorragias por heparina en el tratamiento del tromboembolismo venoso. Angiología 1990;42(1):11-15.

35. Mikkola KM, Patel SR, Parker JA, Grodstein F, Goldhaber SZ. Increasing age is a major risk factor for hemorrhagic complications after pulmonary embolism thrombolysis. Am Heart J 1997;134(1):69-72.

36. The urokinase pulmonary embolism trial (UPET). A national cooperative study. Circulation 1973;47(2 Suppl):II1-130.

37. Meyer G, Gisselbrecht M, Diehl JL, Journois D, Sors $\mathrm{H}$. Incidence and predictors of major hemorrhagic complications from thrombolytic therapy in patients with massive pulmonary embolism. Am J Med 1998;105(6):472-477.

38. Kanter DS, Mikkola KM, Patel SR, Parker JA, Goldhaber SZ. Thrombolytic therapy for pulmonary embolism. Frequency of intracranial hemorrhage and associated risk factors. Chest 1997;111(5):1241-1245.

39. Tibbutt DA, Davies JA, Anderson JA, et al. Comparison by controlled clinical trial of streptokinase and heparin in treatment of life-threatening pulmonary embolism. Br Med J 1974;1(5904):343-347.

40. Dalla-Volta S, Palla A, Santolicandro A, et al. PAIMS 2: alteplase combined with heparin versus heparin in the treatment of acute pulmonary embolism. Plasminogen activator Italian multicenter study 2. J Am Coll Cardiol 1992;20(3):520-526.

41. Nauffal MD, Modesto AM, Ansótegui BE. Diagnóstico y tratamiento del tromboembolismo pulmonar hemodinámicamente inestable. En: Juretschke MMA, Barbosa AC, editores. Tromboembolismo pulmonar. Monografías NEUMOMADRID. Vol. IV. España: NEUMOMADRID, Doyma; 2002. pp. 131-148.

42. Leeper KV Jr, Popovich J Jr, Lesser BA, et al. Treatment of massive acute pulmonary embolism. The use of low doses of intrapulmonary arterial streptokinase combined with full doses of systemic heparin. Chest 1988;93(2):234-240. 
43. Mackman N. Triggers, targets and treatments for thrombosis. Nature 2008;451(7181):914-918.

44. Raschke RA, Gollihare B, Peirce JC. The effectiveness of implementing the weight-based heparin nomogram as a practice guideline. Arch Intern Med 1996;156(15):16451649.

45. Torbicki A, Perrier A, Konstantinides S, et al. ESC Committee for Practice Guidelines (CPG). Guidelines on the diagnosis and management of acute pulmonary embolism: the Task Force for the Diagnosis and Management of Acute Pulmonary Embolism of the European Society of Cardiology (ESC). Eur Heart J 2008;29(18):2276-2315.
46. Stein PD, Hull RD, Raskob G. Risks for major bleeding from thrombolytic therapy in patients with acute pulmonary embolism. Consideration of noninvasive management. Ann Intern Med 1994;121(5):313-317.

\section{$\bowtie$ Correspondencia:}

Dr. Limberth Machado Villarroel, Hospital Ángeles. Av. Campos Elíseos No. 9371, Fracc. Campos Elíseos C.P. 32420, Cd. Juárez, Chihuahua, México. Correo electrónico: limberthmv@hotmail.com Los autores declaran no tener conflictos de interés. 